\title{
Grasping the dynamics of creative play
}

\section{For Early Years Educator}

Authors: Lorna Arnott and Pauline Duncan

\section{Introduction}

In this article we give a brief overview of research findings presented in a recent paper Exploring the Pedagogic Culture of Creative Play in Early Childhood Education available in the Journal of Early Childhood Research. The central focus of the journal article is on children's creative play but from the perspective that the world is complex and cannot neatly be compartmentalised. From that perspective, we present an understanding about how the interlinking elements of a particular context shape how children's creative play presents itself. We do not suggest that there is a typology or framework to follow which will result in children's creativity. Rather we suggest that creativity is highly context specific and even small changes in the context will shift children's creative experience. We say this from a positive stance because it provides opportunities for children's creative expression to flourish in a multitude of different ways and provides practitioners opportunities to facilitate various aspects of creative play by considering the composition of the pedagogic culture.

We were interested in two questions:

1. What are the characteristics of children's creative play across pedagogic cultures?

2. In what ways does the pedagogic culture - consisting of spaces, materials and interpersonal collaborations - shape children's creative play?

\section{Defining Creativity in Education}

Defining creativity is complex and differs across disciplines. Some argue that creativity is an, "imaginative activity fashioned so as to yield an outcome that is of value as well as original", (National Advisory committe on Creative and Cultural Education, 1999, p. 29). The focus then becomes open to interpretation in what is considered an 'outcome', 'of value' and 'original'. In educational contexts the focus is often on developing creativity through imagination and creative thinking (Craft, Jeffrey, \& Leibling, 2001). Consequently, in general creativity is considered a subjective process because understandings of it are often socially constructed (Marsh, 2010).

In our research we focus on a process orientated exploration of creativity. To make this distinction workable with our data, we found it useful to draw on Craft's (2002) distinction between Little C and Big C Creativity. The former predominantly relates to 'process', the child's ability to engage in possibility thinking and ask 'what if' questions; while the latter moves towards the focus on original and unique knowledge and outputs.

What is clear from our data is that creativity, in Education, is considered to be the interplay between 'novelty' and 'usefulness' (Leggett, 2017). In early childhood Education, the focus is often on creative processes, for instance, although construction of some kind may be encouraged, the 
children are not assessed on the quality of the output produced but their learning journey leading up to its production. This approach to creativity marries with typical early childhood pedagogical principles which encourage child-centred and child-led learning experiences. Within this context, the exploration of pedagogic cultures in a holistic manner provides a useful frame to understand the ways in which creative play may manifest.

\section{What is a Pedagogic Culture?}

The article we refer to explores how the pedagogic culture within which children are playing frames creative play, define as:

"an explicit conceptualisation, recognition and application of the ecological elements that frame the

$$
\text { practice around play-based learning in early childhood". }
$$

To understand this perspective, it is important to view play as something which is dynamic and context specific. Play is not a discrete activity but rather the projection, development and cognitive/metacognitive demonstration of children's appropriation of their particular cultural context. That is to say that play is more than simply the resources with which the child engages or the child's spontaneous activity. Play is unique to each context because it is the synergistic output of a specific pedagogic culture. As part of a pedagogic culture, play is the culmination of carefully considered framing. It is this conceptual understanding of early childhood education as something greater and broader than 'play-based pedagogy' that underpins the findings. We need to see children's play as a multi-faceted dynamic endeavour, which evolves alongside society, thinking and cultures.

It is therefore important to recognise that children's creative play is largely influenced by the lived experience in that particular context. That lived experience is a cultural one where children become members of the community. The ways of being in a particular space shape the child's understanding of what is possible. This holistic understanding of children playing within a pedagogical culture which is unique and constantly in flux is the basis for our analysis of the data.

\section{Why we focus on Materials, Interpersonal Collaborations and Space?}

The research questions presented above indicate our interest in three specific elements of a pedagogic culture (there may be more, of course). We suggest that planning for play experiences as situated within a pedagogic culture is as a multi-dimensional task in which the pedagogue must consider, at very least, the use of

(1) space,

(2) interpersonal collaborations as well as

(3) toys and resources (materials).

In doing so the pedagogue scaffolds the child's experiences. We focus on these three areas because they often represent the key influences within an early childhood context. For example, we know from research that space, aesthetics and the division of play areas, influence children's learning experiences (Apps and MacDonald, 2012). We know that furnishings, wall colours and ceiling height impact on behaviour (Read et al. 1999). This information is taken into consideration when designing contemporary nursery buildings. 
Similarly, interpersonal collaborations and interactions are a fundamental focus for all child development. There are well-founded discussions available about the importance of interpersonal interaction in learning, which was somewhat propelled in importance by Vygotsky's Zone of Proximal Development (1978) and then by Wood et al.'s (1976) notion of Scaffolding - the idea that children can learn more with the help of an expert other. In early childhood, we begin to see what this scaffolding may look like in discussions of adult-led and child-led activities (Fisher, 2013), or through adult-structured versus free-choice play approaches to learning. So for us it was interesting to see if these different types of interactions influenced the types of creativity that we observed.

Finally, materials unpin the child's space for learning and in contemporary times the appropriateness of toys is increasingly debated. The evolution of children's toys and resources in modern society brings about discussions amongst childhood educators, parents and the media about 'appropriate' play practices and resources for young children's creative development. The debates are embedded in scholarly explorations and public moral panics about how children's play is being re-evaluated and re-framed as part of digital, multi-modal or contemporary childhoods (e.g. Yelland and Gilbert, 2017). The discussion can be located in considerations of cultural capital and habitus as older generations grew up with markedly different experiences with their childhood toys, creating anxiety over the role of contemporary toys in early childhood (Aldhafeeri et al., 2016). So it seemed helpful to consider the role of resources in relation to children's creative play.

In the article we try to bring all of these elements together to understand how different combinations of space, interpersonal collaborations and materials shaped children's creative expressions.

\section{Three Stories of Creative Play}

This study used data collected from a local nursery and a local science museum. These research sites provided two contrasting spaces to explore children's creativity. The nursery environment was vibrant, noisy and rich in materials (in addition to those provided by the research team). The childled and child-initiated pedagogical approach of the context meant that the number of children in each room continually changed varying from two children up to 10 in one instance. In contrast, we used a closed room in the local science museum and it was quiet, with little distraction. Few resources, other than the ones provided by the researchers, were available.

The context for understanding creativity in the study was children's construction play because practical application is a means of fostering creativity. In both settings, the researchers brought a variety of open-ended and closed-structured construction resources to explore children's creativity as part of their everyday play. Resources included: (i) traditional Froebelian construction blocks (ii) natural tree logs, (iii) scarfs and loose pieces of fabric, (iv) two sets of plastic colourful gears that could be assembled in a variety of interlocking ways, and when constructed 'correctly' could be remote controlled, (v) Meccano Motorised Box - a set of individual pieces which could be assembled into remote controlled vehicles.

We classified these resources as either open-ended materials (if they resembled loose parts play) or closed-structured resources. The closed-structured resources offered a degree of framing for the children because they were presented in boxes with manuals to build a specific output - such as a Ferris Wheel. Children were not instructed by researchers to follow the instructions but certainly the presence of an instruction manual and image of a completed construction had some impact on children's creative thinking, or at least their interpretation of what was required during the play. 
To demonstrate the complexity of children's creative play, we present data from the museum and the nursery, in the form of 'stories'. In each story we present a different variation of the pedagogic culture - relating to space, materials and interpersonal collaborations.

\section{Story 1 - Possibility Thinking and Little C Creativity}

Story One offers a discussion of children's creativity related to possibility thinking and Little C creativity - the process driven creativity. The context within which this creative play emerged was when children engaged in child-child interpersonal collaborations in the nursery space with the open-ended materials. Here, children's creativity was not constrained by any structure imposed by the toys or by other adults. The story demonstrates that this combination of a lively and vibrant environment filled with peers and dynamic interactions with spaces, materials and people results in a pedagogic culture where we observe high levels of possibility thinking and Little C creativity. The children were focused on creative processes rather than pre-planned novel products or outcomes.

Pedagogic Story 1: Possibility Thinking and Little C Creativity

The first story of pedagogic culture related to the synergy of child-child interactions, in the nursery with open-ended materials resources. The free-choice pedagogic principles that underpinned practitioner planning and child-centred play, meant that many children were present in this play space at one time. The result was fleeting engagements with a resource and transitory play themes.

Children moved on from one activity very quickly, and were drawn away to different activities by invites from other children. They rarely produced a preplanned build. Instead they found enjoyment in the exploration phases of creative play, which evolved and changed quickly. Less emphasis was placed on the structured approaches or outcomes and more emphasis was assigned to the play processes and themes. In other words, the children found a place for the resource within their imaginative play theme, rather than finding a theme around the resource. The scaffolding came from a pedagogic culture in the form of freedom of discovery, multiple interactions, opportunities for exploration and trial and error, facilitated by free movement of children and resources.

Blocks offered a freedom that the motorised construction resources did not. Indeed, a case study specifically focusing on early years settings highlighted that formless materials can be conducive to creativity rather than more prescriptive resources favoured by older children. Our observations suggest this is due to both familiarity with open-ended materials blocks and their simpler or less prescribed form children quickly and efficiently constructed towering structures. They also do not 'connect' in specific ways but could be successfully stacked and combined in endless ways as shown in Figure 2.

Figure 1: Endless possibilities

\section{Story 2 - Problem Solving and Novel Output (Bigger C Creativity).}

Story Two describes what we have labelled Bigger $C$ creativity, to reflect the focus on production of outputs and products. We argue that this is better defined as 'Bigger $C^{\prime}$ creativity to reflect that this is still a learning process for children and not a finished product. This dimension of creative play 
appeared to be facilitated by a pedagogic culture instilled with relaxation, free mobility and access to resources, Sustained Shared Thinking in relation to a planned build or task, and problem solving. All of which focused around perceived affordances of the resources, combined with time for quiet contemplation and the presence of an expert other. Story 2, therefore, offers a discussion of adultchild interpersonal collaborations, in the museum space with closed-structured construction materials.

Pedagogic Story 2: Problem Solving and Novel Output (Bigger C Creativity).

In this story of pedagogic culture, we explore the intersection of closed-structured construction resources, in a tranquil museum context with adult-child interactions. The children were free from external distractions and only influenced by their parents. In all but one case, parents were happy for children to remain in the play space for as long as the children wished (sometimes lasting up to one hour). All of these things combined resulted in more sustained engagements, over time. The children focused on creative outputs in the sense that children actually verbalised a pre-planned idea for a construction, followed through and completed what they set out to build. The process was more structured and methodological, relating to completion of the task, and focused on fostering structured uses of resources which were linked closely to their affordances and the resources intended purpose.

In almost all cases, the resource became the central focus of the play. It became a task that should be completed before moving on to something else. In some cases, adults directed the play, in others the child took the lead - but in almost all cases, there was an understanding that they were playing within the confines of the affordances of the resource, such as building a fair ground with the motorised closed-structured construction toys. In only one instance of adult-child interaction did they engage in transgression - where they transformed objects into another meaning - and use the resources in unconventional ways. Here the activity was transformed into a role play scenario where the Meccano was used as a doctors kit and petrol pump. This evolution of play focus was initiated by the child. There were no instances of adults initiating a suggestion that resources could be used in any way other than their intended purpose.

\section{Figure 2: Mother and Daughter exploring the closed-structured construction} resources

\section{Story 3 - Foundations to Creative Play}

Pedagogic Story 3 presents a reflection on the kinds of pedagogic planning required in order to foster creative play. Children need time to engage with the resources - deconstructing and manipulating resources to learn about their affordances - before creative play can evolve and progress. This dimension of creative play appeared to be facilitated by a pedagogic culture inherent with challenge and difficulty. This was because the materials were complex and required knowledge of the mechanical working of the cogs as well as logical planning in order to correctly assemble the 
pieces. As such this pedagogic culture instilled a sense of novelty and of the unknown, as children were left to discover amongst themselves in a child-led, free choice space.

Story Three therefore offers a discussion of child-child interpersonal collaborations, in the nursery space with closed-structured construction materials. It demonstrated that this synergy of pedagogic culture offers routes to exploration and tinkering; yet another manifestation of creative play.

\section{Story 3: Foundations to Creative Play}

The third story of pedagogic culture describes the intersection of child-child interactions, with closed-structured construction toys in the nursery context. Children were less familiar with the resources and the children were quick to abandon the resource and move onto another activity. This complexity arises from the difficulties inherent with planning and creating resources with which one is unfamiliar. This was primarily due to that fact that motorised playsets are idiosyncratic with intricate, complex features that vary across brands. For this reason, exploration is essential in order to familiarise oneself with new or more complex creative tools. Children tested the ways in which pieces fit together, explored how pieces could be deconstructed, and manipulated parts by pressing buttons, pulling the spring-like features, and playing with the fibre optic strands.

Figure 3: Exploration of closed-structured construction toys

As a consequence, the children gained an understanding of what each button and resource did, and how they could manipulate each object for their desired creative purposes. It is only once the knowledge and understanding of the resources' affordance is acquired can the child achieve higher levels of creativity.

Emily says the robot isn't working because only one cog is turning. She presses the button on the remote....She adds some more cogs to the central moving part and then tests it again using the remote. She spins the other cogs using her other hand as they are still not turning. She then tries to make the robot stand up. It doesn't stand independently. She looks quizzically at it. 'Maybe it's not standing because it doesn't have legs!?' She then takes some cogs and adds them to the bottom of the structure. Alas, it still does not stand. (Emily playing with construction resources - Nursery observation)

Due to children's unfamiliarity with the affordances of the closed-structured construction toys, they looked to external stimuli to guide their play. External stimuli was required to foster creative outputs and constructions with closedstructured construction toys. They used the handbooks or looked at the box to understand its potential.

\section{What does this mean for practice: Diversity in creative play}

What we present here are three, unique pieces of evidence of what creativity can look like in early childhood. Our findings suggest that creative play took different forms across pedagogic cultures. 
The data suggests that children do not only engage in one form of creative play in each pedagogic culture, but rather that each pedagogic culture in our study supported different manifestations of creative play at that particular snapshot in time. The nature of children's creative play varied depending upon the composition and framing of the pedagogic culture as shaped by the three central elements - space, interpersonal collaborations and materials.

In terms of this data being meaningful in practice, we suggest that - despite not trying to prescribe or manufacture creative experience - practitioners are in a position to facilitate various aspects of creative play by considering or tweaking the make-up of the pedagogic culture. For example, reflecting on how the interpersonal collaborations (i.e. the adult or child's ability to scaffold the creative play in times of need), the materials (the challenge and familiarity associated with the resource) and the spaces (creating a space for concentration versus high energy exploration) all influence children's thinking, engagement and interactions during the creative process. We know from Reggio Emilia that the environment is considered the third teacher. Here we suggest that for creative play, the specific framing of the pedagogic culture acts as a facilitator in creating opportunities for varied and unique explorations of creativity to emerge and be valued.

We must consider how these elements intertwine within a multidimensional pedagogic culture to create new manifestations of creative play. Just as the children need time to explore and become familiar with the affordances of the resource to engage in higher levels of creative play, so to do we need to become familiar with the impact of a specific pedagogic culture through observation and analysis. This requires thoughtful consideration of observations over time to reflect on the nuanced differences in children's manifestations of creative play to understand what can be put in place to enhance the creative play going forward.

The differentiated manifestations of creative play across pedagogic cultures become particularly important when making sense of our data because they provide a frame for questioning pedagogic planning. We do not present a typology that guarantees a specific manifestation of creativity, if replicated. Our epistemological stance stems from co-constructivist thinking, and suggests that children never experience a pedagogic culture in a unified manner. Children's interpretations and reproduction of the culture will shape the manifestations of creative play that emerge. Thus we advocate for a culture where time and freedom to explore is paramount; not only for children but for adults in the setting too.

The model is therefore not directive, but presents an understanding of some of the major contextual and cultural factors which practitioners could draw on to begin to understand how creative play may manifest in practice. In essence, we see creative play as a dynamic, multi-faceted and relational process, shaped by the pedagogic culture.

\section{References}

Aldhafeeri F, Palaiologou I and Folorunsho A. (2016) Integration of digital technologies into playbased pedagogy in Kuwaiti early childhood education: teachers' views, attitudes and aptitudes. International Journal of Early Years Education 24: 342-360.

Apps L and MacDonald M. (2012) Classroom aesthetics in early childhood education. Journal of Education and learning 1: 49.

Craft, A., Jeffrey, Bob, \& Leibling, Mike. (2001). Creativity in education: Continuum. 
Craft A. (2002) Creativity and early years education: a lifewide foundation: Continuum.

Fisher J. (2013) Starting From The Child: Teaching And Learning In The Foundation Stage: Teaching and Learning from 4 - 8, Maidenhead: McGraw-Hill Education.

Leggett N. (2017) Early Childhood Creativity: Challenging Educators in Their Role to Intentionally Develop Creative Thinking in Children. Early Childhood Education Journal.

National Advisory committee on Creative and Cultural Education. (1999). All Our Futures: Creativity, Culture and Education.

Read, M, Sugawara, A, \& Brandt, J. (1999). Impact of Space and Color in the Physical Environment on Preschool Children's Cooperative Behaviour. Environment and Behavior, 31(3), 413-428

Vygotsky, L. S. (1978). Mind in society : the development of higher psychological processes. Cambridge: Harvard University Press.

Wood D, Bruner JS and Ross G. (1976) The role of tutoring in problem solving. Journal of child psychology and psychiatry 17: 89-100. 